\title{
Prestige Matters: Wage Premium and Value Addition in Elite Colleges
}

\author{
By SHEETAL SEKHRI*
}

\section{Online Appendix : Not for Publication}

\section{Data Appendix}

Admission data linked to university wide college exit test scores was available for 5 cohorts. The original addresses for students from their application forms along with a phone number were obtained from the colleges. However, the addresses for the oldest cohort were not available so I did not survey this cohort. The sample frame for the survey included graduates for whom I also had exit tests final scores in the university exam. Because of cost of survey and tracking revisits, the frame was restricted to urban areas only. Rural students were not interviewed. This survey was also not administered to anyone who was admitted to the colleges on a reserved seat. ${ }^{1}$ Target list was everyone for whom we had an address and or a phone number.

The tracking system was quite onerous and expensive. We made contact over the phone to ascertain if the resident knew the respondent. But a lot of phone numbers had changed. So we made a first visit to the household to verify the addresses. In majority of cases, we were able to verify the residents either lived at the address or a family member/ neighbor was able to provide a contact address. Subsequently, we visited the households and tried to conduct the survey. The revisit rate was high as this population is employed and was not readily available for interviews. We set a maximum of 3 visits to conclude the surveys. If we did not get a response by the third visit, we did not revisit the respondent. Median cases were visited twice.

On comparing the admission characteristics of the individuals successfully surveyed (matched) versus those who were not (unmatched), I observe several differences in the full sample. However, these samples are comparable in a narrow interval of -5 to +5 points around the threshold. Individuals whose father's were employed in service in formal sector were marginally less successfully surveyed. This may be because of transferable nature of the jobs. The difference is small. Science graduates were less successfully

\footnotetext{
* Sekhri: University of Virginia, PO Box 400182, Department of Economics, Monroe Hall, Charlottesville, VA 229044182, USA, Email: SSekhri@ virginia.edu, Phone:434-982-4286,Fax:434-982-2904. Funding from International Growth Centre, India Country Team (CPP-IND-CEN-2010-008) is greatly acknowledged. Zhou Zhang and Sisir Debnath provided excellent research assistance. This paper has benefitted from discussions with Ken Chay, Andrew Foster, Leora Friedberg, Claudia Goldin, Kevin Lang, John Pepper, Sarah Turner, Miguel Urquiola, Eric Chyn, Amalia Miller, John Pepper, Harald Tauchmann and from suggestions of seminar participants at Boston University and IGC Growth Week.

${ }^{1}$ There is reservation for lower caste students, victims of riots, children or grand children of freedom fighters, and non reserved candidates.
} 
surveyed albeit the difference is again small. Any bias can only result if these characteristics are different by colleges types in a narrow margin around the cutoff. This is addressed in the manuscript in subsection 6.1.

\section{Procedure Details for the Lee Bounds}

I compute a trimmed mean for the private colleges trimming the distribution in the right and the left tails by an amount equal to the difference in these shares. Evaluating the difference of the mean of the public colleges and these trimmed means gives us the lower and upper bounds respectively. The bounds for the 5 point interval are calculated analogously and reported in column (ii) of Appendix Table 9.

In the full sample, the fraction with missing value are 0.329 and 0.454 in private and public colleges respectively. The difference in shares is 0.1865 . This implies that the private college mean will be trimmed by the trimming share equal to 0.1865 . Hence, 49.806 highest and lowest observations from the private college distribution have to be excluded to calculate the trimmed mean. The trimmed average of salary for the private colleges with lowest values excluded is 19205.4 and the highest value excluded is 13099.08. Now taking the difference of the mean of the public colleges and these trimmed means gives us the lower and the upper bounds of 6147.7 and 12254.3 respectively. These trimmed means use 49 highest and lowest values. If we use 49 lowest and highest values with a weight of 1 and the 50th value with a weight of 0.194 (that is, the difference between 50 and 49.806) for the calculation of the trimmed means, we get the lower bound of $6,158.8$ and an upper bound of 12,243.6 rupees both significant at the 1 percent significance level. The bounds for the 5 point interval are calculated analogously and reported in column (ii). ${ }^{2}$

\section{REFERENCES}

Lee, David S. 2009. "Training, wages, and sample selection: Estimating sharp bounds on treatment effects." The Review of Economic Studies, 76(3): 1071-1102.

Tauchmann, Harald. 2014. "Lee (2009) treatment-effect bounds for nonrandom sample selection.” The Stata Journal, 14(4): 884-894.

\footnotetext{
${ }^{2}$ This exercise has been done using Lee's bound STATA module (Lee 2009). I thank Harald Tauchman (Tauchmann 2014) for extensive discussion about this module.
} 
APPENDix Figures \& TABLES

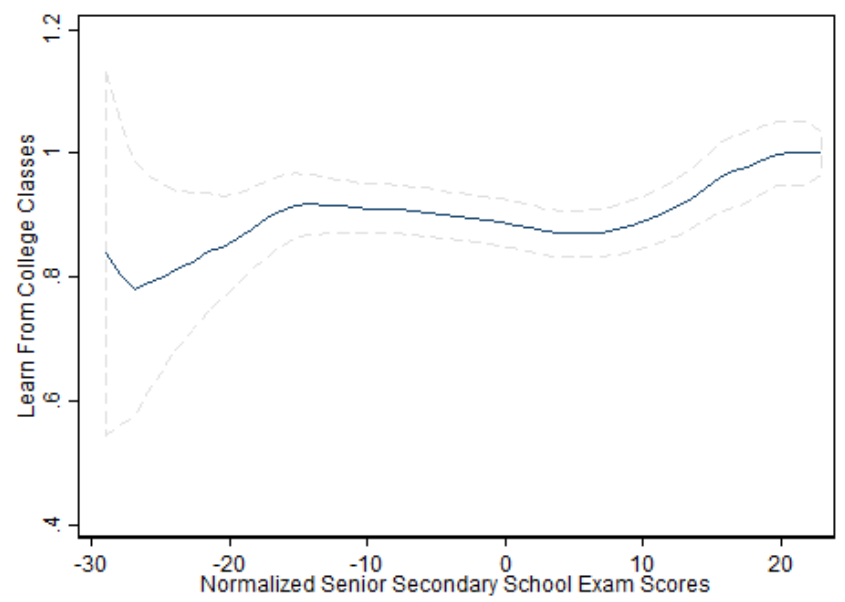

Figure A1. Self -Reported Assessment of Learning from College Classes 


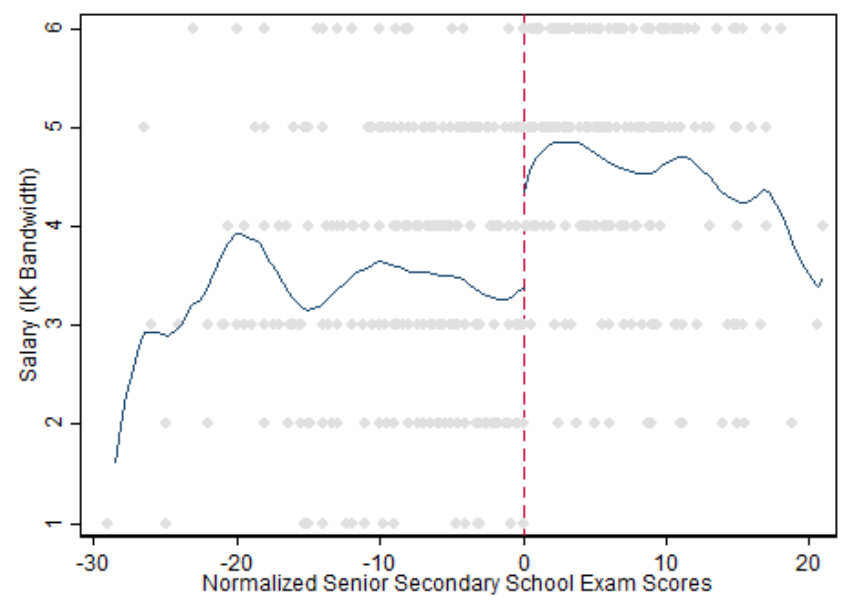

Figure A2. Discontinuity in SAlary at the Public College Admission Cutoff (IK Bandwidth)

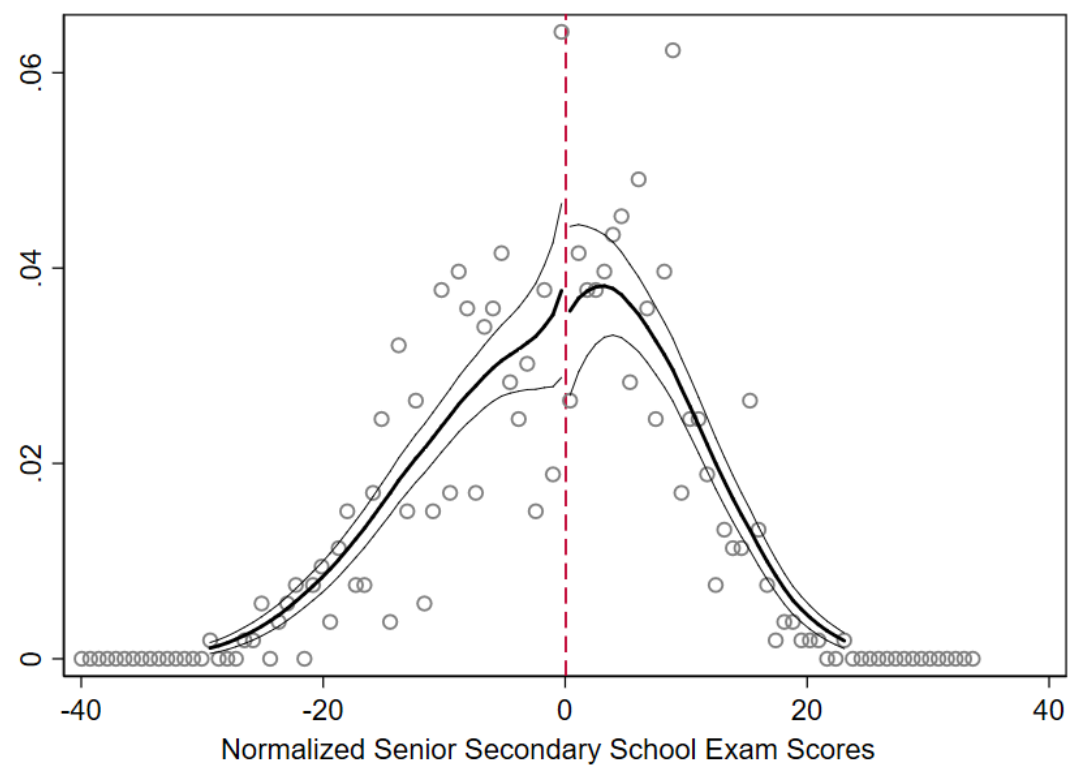

Figure A3. Smooth Density of Forcing VARIABle 

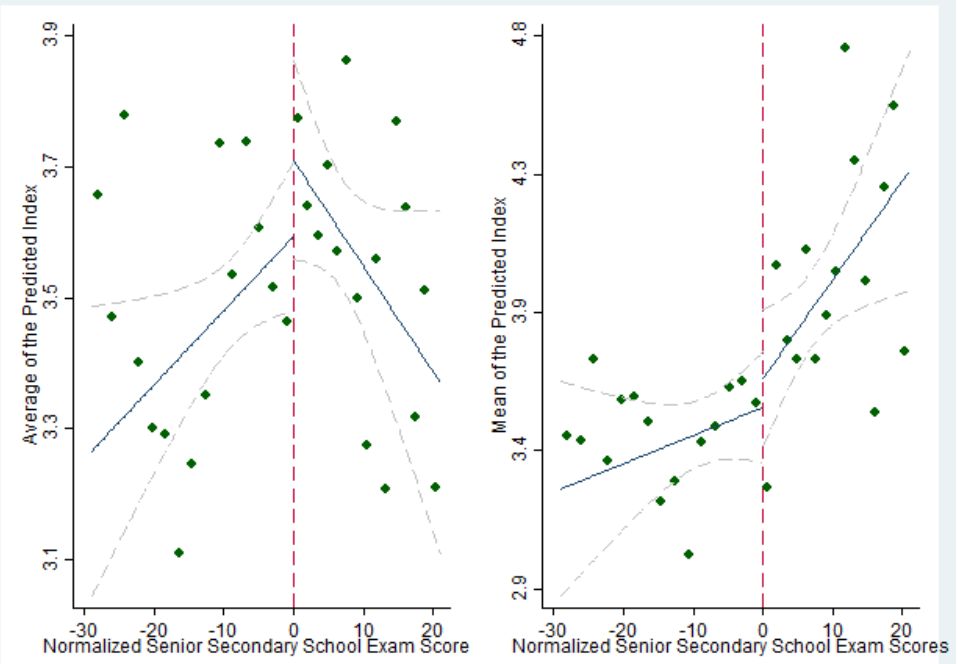

Figure A4. CONTINUITY in BACKgRound CHARACTERISTICS

Note: Index is predicted salary based on a regression of salary on background covariates in a sample restricted to private colleges in Panel A and in a sample outside the -15 to +15 points intervals of the Normalized Senior Secondary Exam scores in Panel B. The figures plot the average value of the index by bins of normalized Senior Secondary School Exam Scores along with the line of best fit and confidence intervals.

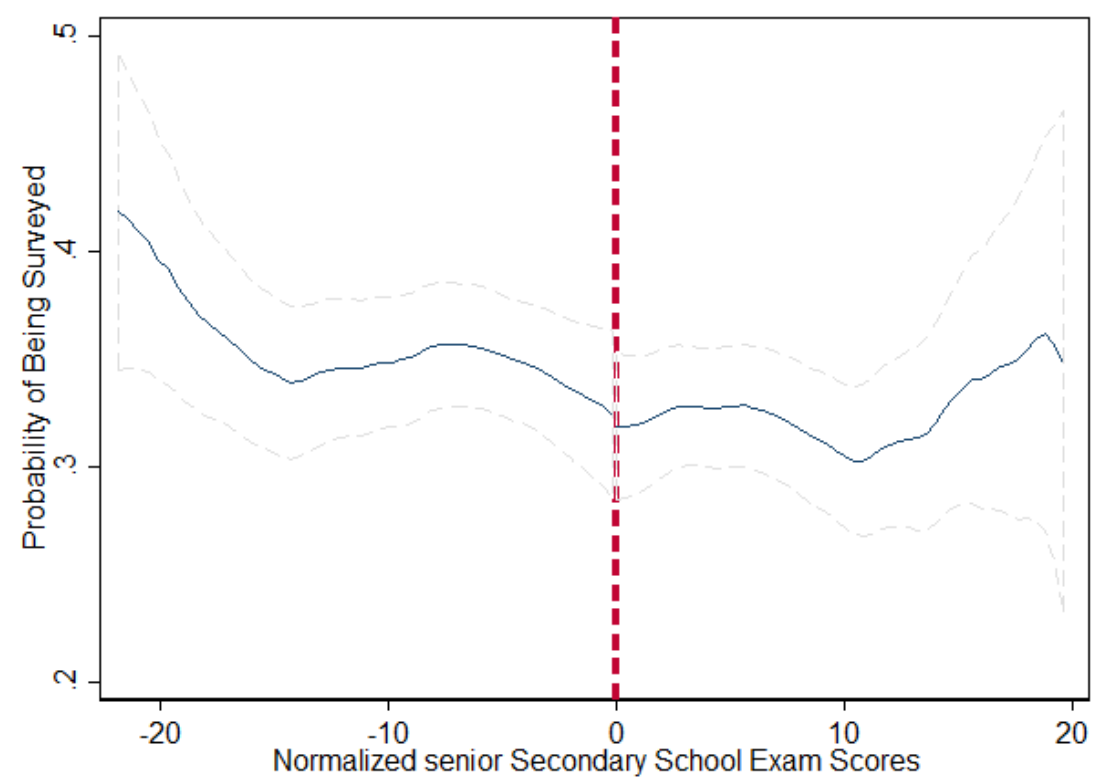

Figure A5. Probability of Survey Success 


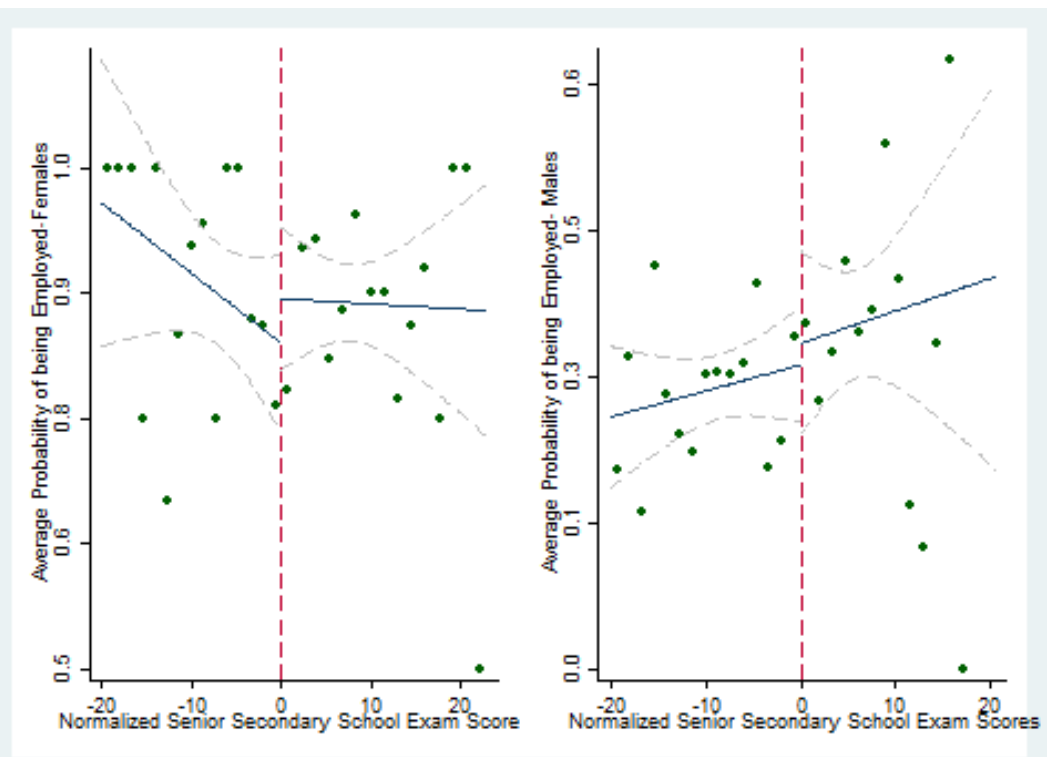

Figure A6. Probability of BeIng Employed

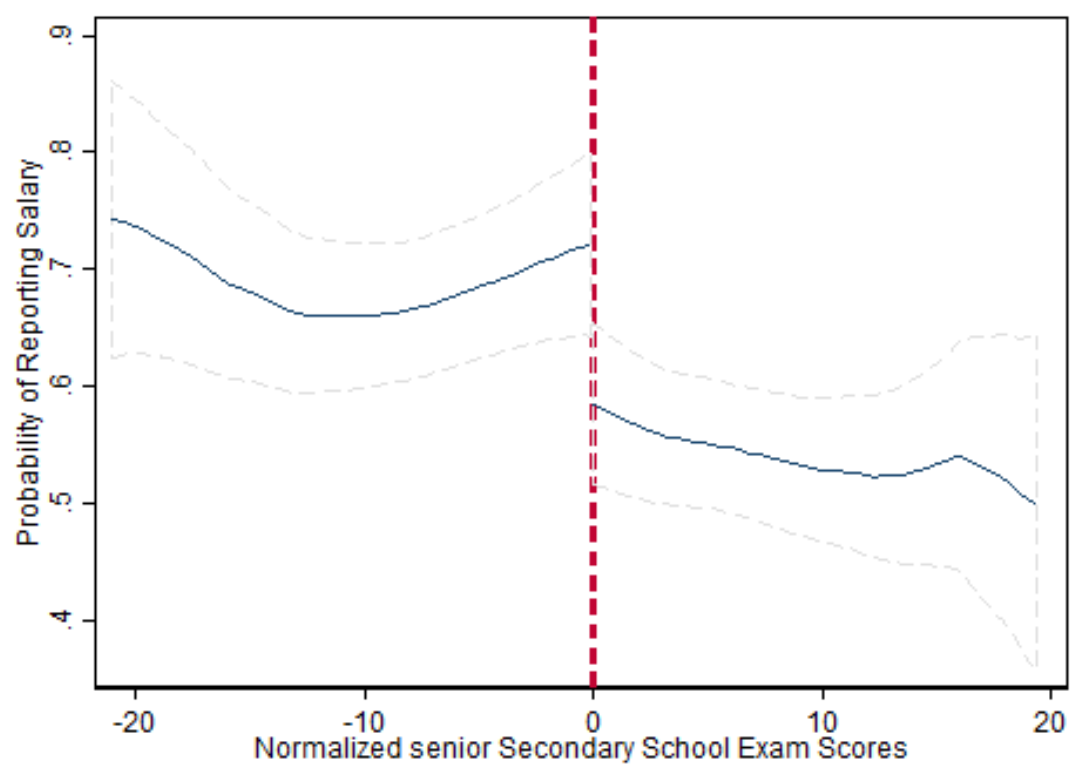

Figure A7. DiscontinUity in PRobability of Reporting SALARY (IK BANDWidTh) 
Table A1-Summary Statistics by College Type in -5 to 5 Interval of Senior Secondary School NORMALIZED SCORES

\begin{tabular}{|c|c|c|c|c|c|c|}
\hline & & \multicolumn{2}{|c|}{ Public } & \multicolumn{2}{|c|}{ Private } & \multirow[b]{2}{*}{ Difference } \\
\hline & & mean & std dev & mean & std dev & \\
\hline \multicolumn{2}{|l|}{ Salary } & 5.00 & 1.00 & 3.55 & 1.44 & 1.36 \\
\hline \multicolumn{2}{|c|}{ Senior Secondary School Exam Scores } & 70.40 & 8.00 & 67.26 & 7.40 & 3.20 \\
\hline \multicolumn{2}{|c|}{ Central Board of Secondary Education } & 0.22 & 0.42 & 0.21 & 0.41 & 0.01 \\
\hline \multicolumn{2}{|c|}{ Age at Starting College } & 18.00 & 0.91 & 18.00 & 0.90 & 0.08 \\
\hline \multicolumn{7}{|c|}{ Father's Occupation } \\
\hline & Government Service & 0.10 & 0.30 & 0.09 & 0.28 & 0.01 \\
\hline & Labor in Unorganized Sector & 0.03 & 0.02 & 0.06 & 0.02 & 0.03 \\
\hline & Professional & 0.06 & 0.24 & 0.05 & 0.23 & 0.01 \\
\hline & Service in Formal Sector & 0.35 & 0.48 & 0.23 & 0.42 & 0.11 \\
\hline & Agriculture & 0.08 & 0.27 & 0.09 & 0.29 & 0.01 \\
\hline & Business & 0.25 & 0.43 & 0.32 & 0.47 & 0.07 \\
\hline \multicolumn{7}{|c|}{ Admission Year } \\
\hline & 1999 & 0.20 & 0.40 & 0.26 & 0.44 & 0.05 \\
\hline & 2000 & 0.37 & 0.48 & 0.19 & 0.40 & 0.18 \\
\hline & 2001 & 0.30 & 0.45 & 0.26 & 0.44 & 0.03 \\
\hline & 2002 & 0.12 & 0.33 & 0.28 & 0.45 & 0.15 \\
\hline Male & & 0.46 & 0.50 & 0.35 & 0.05 & 0.11 \\
\hline \multicolumn{7}{|l|}{ Stream } \\
\hline & Commerce & 0.27 & 0.45 & 0.30 & 0.45 & 0.016 \\
\hline & Liberal Arts & 0.51 & 0.50 & 0.45 & 0.5 & 0.06 \\
\hline & Science & 0.20 & 0.40 & 0.25 & 0.43 & 0.047 \\
\hline \multicolumn{2}{|c|}{ College Exit Test Scores } & 1272.35 & 276.00 & 1247.00 & 271.20 & 25.27 \\
\hline \multicolumn{2}{|l|}{ Observations } & 79 & & 88 & & \\
\hline
\end{tabular}


TABle A2-Non-Parametric RDD Estimates of the EFFect of Public Colleges on SALARY

\begin{tabular}{|c|c|c|c|c|}
\hline \multicolumn{5}{|c|}{ Dependent Variable: Reported Salary (Midpoint of the Bins for the Categorical Variable) } \\
\hline Panel B: Non-Parametric Estimates & $\begin{array}{c}\text { Bandwidth } 10 \\
\text { (i) }\end{array}$ & $\begin{array}{c}\text { Bandwidth } 7.5 \\
\text { (ii) }\end{array}$ & $\begin{array}{l}\text { Bandwidth } 5 \\
\text { (iii) }\end{array}$ & $\begin{array}{c}\text { Bandwidth IK } \\
\text { (iv) }\end{array}$ \\
\hline Kernel Triangle & $\begin{array}{l}10727.4 \\
(2299.1)\end{array}$ & $\begin{array}{l}17476.44 \\
(4310.5)\end{array}$ & $\begin{array}{l}17753.8 \\
(5855.8)\end{array}$ & $\begin{array}{l}15340.3 \\
(2756.8)\end{array}$ \\
\hline Kernel Rectangular & $\begin{array}{l}16668.4 \\
(3015.8)\end{array}$ & $\begin{array}{l}16336.0 \\
(3575.5)\end{array}$ & $\begin{array}{l}18946.3 \\
(4789.6)\end{array}$ & $\begin{array}{l}11035.62 \\
(2027.5)\end{array}$ \\
\hline
\end{tabular}

Notes: Demographic controls included are gender, year of admission in college, age at entering college, stream of study, board of education for Senior Secondary school, and father's occupation. Robust standard errors are reported in parenthesis. Public College is an indicator equal to 1 if the individual attended public college and 0 if the individual graduated from a private college.

TABle A3-Survey Success by College Type in 5 Point Interval

\begin{tabular}{|c|c|c|c|c|c|}
\hline \multirow[b]{2}{*}{ Variable } & \multicolumn{2}{|c|}{ Public } & \multicolumn{2}{|c|}{ Private } & \multirow{2}{*}{$\begin{array}{l}\text { Equivalence Test } \\
\text { p-value }\end{array}$} \\
\hline & Coefficient & Standard Error & Coefficient & Standard Error & \\
\hline Commerce & 0.08 & 1.50 & 0.06 & 0.91 & 0.51 \\
\hline Science & -0.03 & 0.07 & 0.01 & 0.06 & 0.66 \\
\hline Age at Entering College & -0.02 & 0.03 & 0.02 & 0.03 & 0.33 \\
\hline Father Skilled Profession & 0.08 & 0.10 & -0.04 & 0.09 & 0.35 \\
\hline Father in Agriculture & 0.18 & 0.10 & -0.05 & 0.09 & 0.09 \\
\hline Father in Business & 0.18 & 0.05 & 0.03 & 0.04 & 0.02 \\
\hline Admission Year 1999 & 0.09 & 0.06 & 0.00 & 0.05 & 0.27 \\
\hline Admission Year 2000 & 0.11 & 0.05 & 0.11 & 0.06 & 0.96 \\
\hline
\end{tabular}

Notes: Dependent variable 'survey success' takes value 1 if individual was successfully surveyed and 0 otherwise. Columns (i) and (iii) report the coefficients from the regression of 'survey success' on background characteristics for public and private colleges respectively. Columns (ii) and (iv) report the standard errors from these regressions. Column (v) reports the p-value from the test of equivalence of the regressors. Public College is an indicator equal to 1 if the individual attended public college and 0 if the individual graduated from a private college. 
Table A4-Parametric RD Estimates of the EfFect of Public Colleges on being Employed

\begin{tabular}{lcccc}
\hline \multicolumn{5}{c}{ Dependent Variable: Self Employed or Working for Salary } \\
\hline & Full sample & $\begin{array}{c}\mathbf{1 5} \text { Point } \\
\text { Interval } \\
\text { Senior Secondary School Exam Scores }\end{array}$ & $\begin{array}{c}\mathbf{1 0} \text { Point } \\
\text { Interval } \\
\text { (iii) }\end{array}$ & $\begin{array}{c}\mathbf{5} \text { Point } \\
\text { Interval } \\
\text { (iv) }\end{array}$ \\
\hline \hline Linear & 0.05 & 0.06 & 0.03 & 0.06 \\
& $(0.03)$ & $(0.04)$ & $(0.04)$ & $(0.05)$ \\
Quadratic & 0.05 & 0.06 & 0.04 & 0.067 \\
& $(0.03)$ & $(0.04)$ & $(0.04)$ & $(0.053)$ \\
Cubic & 0.04 & 0.06 & 0.03 & 0.05 \\
& $(0.036)$ & $(0.04)$ & $(0.04)$ & $(0.05)$ \\
& & & & \\
\hline Observations & 1505 & 1285 & 1017 & 549 \\
\hline
\end{tabular}

Notes:Demographic controls include gender, year of admission in college, age at entering college, stream of study, board of education for Senior Secondary school, and father's occupation. Robust standard errors are reported in parenthesis. Public College is an indicator equal to 1 if the individual attended public college and 0 if the individual graduated from a private college.

Table A5-Parametric RD Estimates of the EfFect of Public Colleges on Probability of Employment by Gender

\begin{tabular}{lccc}
\hline \multicolumn{4}{c}{ Dependent Variable:Self Employed or Working for Salary } \\
\hline \multirow{4}{*}{$\begin{array}{c}\text { 15 Point Interval } \\
\text { (i) }\end{array}$} & $\begin{array}{c}\mathbf{1 0} \text { Point Interval } \\
\text { (ii) }\end{array}$ & $\begin{array}{c}\mathbf{5} \text { point Interval } \\
\text { (iii) }\end{array}$ \\
\hline \hline \multirow{4}{*}{ Males } & 0.035 & -0.0004 & -0.014 \\
& $(0.051)$ & $(0.06)$ & $(0.07)$ \\
Females & 0.05 & 0.03 & 0.09 \\
& $(0.057)$ & $(0.063)$ & $(0.091)$ \\
& & & \\
\hline
\end{tabular}

Notes: Demographic controls include year of admission in college, age at entering college, stream of study, board of education for Senior Secondary school, and father's occupation. Robust standard errors are reported in parenthesis. Public College is an indicator equal to 1 if the individual attended public college and 0 if the individual graduated from a private college. Linear control function specification is used. 
Table A6-RD Estimates of the EfFect of Public Colleges on Salary by Gender

Dependent Variable: Reported Salary (Midpoint of the Bins for the Categorical Variable)

\begin{tabular}{lccc} 
& $\begin{array}{c}\text { 15 Point Interval } \\
\text { (i) }\end{array}$ & $\begin{array}{c}\text { 10 Point Interval } \\
\text { (ii) }\end{array}$ & $\begin{array}{c}\mathbf{5} \text { point Interval } \\
\text { (iii) }\end{array}$ \\
\hline \hline \multirow{3}{*}{ Males } & & & \\
& 8229.19 & 8457.01 & 10660.85 \\
\multirow{4}{*}{ Females } & $(2850.5)$ & $(3577.1)$ & $(4376.2)$ \\
& & & \\
& 7339.2 & 6485.7 & 6670.4 \\
& $(2179.7)$ & $(2347.8)$ & $(2956.6)$
\end{tabular}

Notes: Each column reports the coefficient from a different regression controlling for year of admission in college, age at entering college, stream of study, board of education for Senior Secondary school, and father's occupation. Robust standard errors are reported in parenthesis. Public College is an indicator equal to 1 if the individual attended public college and 0 if the individual graduated from a private college. Linear control function specification is used. 
TABle A7—Characteristics of INDIVIDUALS REPORTING SALARY VERSUS Not

\begin{tabular}{|c|c|c|c|}
\hline & Salary Reported & Salary not Reported & Difference \\
\hline Senior Secondary School Exam Scores & $\begin{array}{l}66.57 \\
(0.48)\end{array}$ & $\begin{array}{l}66.35 \\
(0.63)\end{array}$ & -0.21 \\
\hline Age at Entering Colleges & $\begin{array}{c}18.03 \\
(0.046)\end{array}$ & $\begin{array}{l}18.15 \\
(0.04)\end{array}$ & 0.12 \\
\hline Admission Year 1999 & $\begin{array}{c}0.21 \\
(0.02)\end{array}$ & $\begin{array}{c}0.26 \\
(0.02)\end{array}$ & 0.04 \\
\hline Admission Year 2000 & $\begin{array}{c}0.29 \\
(0.02)\end{array}$ & $\begin{array}{c}0.30 \\
(0.02)\end{array}$ & 0.01 \\
\hline Admission year 2001 & $\begin{array}{c}0.24 \\
(0.02)\end{array}$ & $\begin{array}{c}0.02 \\
(0.02)\end{array}$ & -0.03 \\
\hline Admission year 2002 & $\begin{array}{c}0.24 \\
(0.02)\end{array}$ & $\begin{array}{c}0.23 \\
(0.02)\end{array}$ & -0.017 \\
\hline Central Board of Secondary Education & $\begin{array}{c}0.31 \\
(0.02)\end{array}$ & $\begin{array}{c}0.25 \\
(0.02)\end{array}$ & 0.06 \\
\hline Male & $\begin{array}{c}0.40 \\
(0.023)\end{array}$ & $\begin{array}{c}0.78 \\
(0.02)\end{array}$ & 0.38 \\
\hline $\begin{array}{l}\text { Father's occupation } \\
\text { Government Service }\end{array}$ & $\begin{array}{c}0.08 \\
(0.01)\end{array}$ & $\begin{array}{c}0.04 \\
(0.01)\end{array}$ & -0.04 \\
\hline Labor in Unorganized Sector & $\begin{array}{c}0.07 \\
(0.01)\end{array}$ & $\begin{array}{c}0.05 \\
(0.01)\end{array}$ & -0.02 \\
\hline Professional & $\begin{array}{c}0.05 \\
(0.01)\end{array}$ & $\begin{array}{c}0.02 \\
(0.008)\end{array}$ & -0.03 \\
\hline Service in Formal sector & $\begin{array}{c}0.30 \\
(0.02)\end{array}$ & $\begin{array}{c}0.25 \\
(0.02)\end{array}$ & -0.067 \\
\hline Agriculture & $\begin{array}{c}0.07 \\
(0.01)\end{array}$ & $\begin{array}{c}0.06 \\
(0.01)\end{array}$ & -0.007 \\
\hline Business & $\begin{array}{c}0.30 \\
(0.02)\end{array}$ & $\begin{array}{c}0.36 \\
(0.03)\end{array}$ & 0.07 \\
\hline $\begin{array}{l}\text { Streams } \\
\text { Commerce }\end{array}$ & $\begin{array}{c}0.24 \\
(0.02)\end{array}$ & $\begin{array}{c}0.35 \\
(0.03)\end{array}$ & 0.1 \\
\hline Liberal Arts & $\begin{array}{c}0.51 \\
(0.02)\end{array}$ & $\begin{array}{c}0.57 \\
(0.03)\end{array}$ & 0.06 \\
\hline Science & $\begin{array}{c}0.24 \\
(0.02)\end{array}$ & $\begin{array}{l}0.06 \\
0.01\end{array}$ & -0.17 \\
\hline
\end{tabular}


Table A8-Parametric RdD Estimates EfFect of Public Colleges on Salary (Imputed)

\begin{tabular}{|c|c|c|c|}
\hline \multicolumn{4}{|c|}{ Dependent Variable: Imputed Salary Conditional on employment } \\
\hline & \multicolumn{3}{|c|}{ (Midpoint of the Bins for the Categorical Variable) } \\
\hline & $\begin{array}{l}15 \text { Point Interval } \\
\text { (i) }\end{array}$ & $\begin{array}{c}10 \text { Point Interval } \\
\text { (ii) }\end{array}$ & $\begin{array}{l}5 \text { Point Interval } \\
\text { (iii) }\end{array}$ \\
\hline $\begin{array}{l}\text { Linear Control } \\
\text { Function }\end{array}$ & $\begin{array}{c}7590.16 \\
(1555.483)\end{array}$ & $\begin{array}{c}7194.5 \\
(1831.61)\end{array}$ & $\begin{array}{l}7612.304 \\
(2069.9)\end{array}$ \\
\hline $\begin{array}{l}\text { Quadratic Control } \\
\text { Function }\end{array}$ & $\begin{array}{c}7726.9 \\
(1621.8)\end{array}$ & $\begin{array}{c}7204.9 \\
(1823.6)\end{array}$ & $\begin{array}{l}7321.8 \\
(2067.2)\end{array}$ \\
\hline $\begin{array}{l}\text { Cubic Control } \\
\text { Function }\end{array}$ & $\begin{array}{c}7826.2 \\
1618.05\end{array}$ & $\begin{array}{c}7200.7 \\
(1759.1)\end{array}$ & $\begin{array}{l}7346.2 \\
(2216.2)\end{array}$ \\
\hline Observations & 629 & 510 & 261 \\
\hline
\end{tabular}

Table A9-Bounded Effect of Public Colleges on Salary Based on LeE’s Bounds

\begin{tabular}{|c|c|c|}
\hline \multicolumn{3}{|c|}{ Dependent Variable: Reported Salary in 6 Categorical Brackets } \\
\hline & Full sample & $\begin{array}{l}-5 \text { to }+5 \text { Interval } \\
\text { around cutoff }\end{array}$ \\
\hline & (i) & (ii) \\
\hline Number of Observations & 748 & 274 \\
\hline Number of Observations with Non Missing Salary & 458 & 171 \\
\hline Trimming Proportion & 0.1865 & 0.1947 \\
\hline Confidence Interval for the Treatment Effect [95 percent] & {$[0.4431 .90]$} & {$\left[\begin{array}{lll}0.503 & 2.3\end{array}\right]$} \\
\hline \multicolumn{3}{|l|}{ Lower Bound } \\
\hline Coefficient & 6158.804 & 7705.6 \\
\hline Standard Error & 1264.5 & $(1973.3)$ \\
\hline \multicolumn{3}{|l|}{ Upper Bound } \\
\hline Coefficient & 12243.6 & 13777.4 \\
\hline Standard Error & $(1187.0)$ & $(1848.4)$ \\
\hline
\end{tabular}

Notes: Both specifications control for year of admission in college, age at entering college, stream of study, board of education for Senior Secondary school, and father's occupation. Robust standard errors are reported in parenthesis. Public College is an indicator equal to 1 if the individual attended public college and 0 if the individual graduated from a private college. Linear control function specification is used. 